\title{
Effect of Cardiac-Cycle-Synchronized Selective Vagal Stimulation on Heart Rate and Blood Pressure in Rats
}

\author{
Dennis T. T. Plachta · Josef Zentner - Debora Aguirre • \\ Oscar Cota $\cdot$ Thomas Stieglitz $\cdot$ Mortimer Gierthmuehlen
}

Received: March 30, 2016/Published online: May 24, 2016

(C) Springer Healthcare 2016

\begin{abstract}
Introduction: Activation of the baroreflex system through the selective vagal nerve stimulation (sVNS) may become a treatment option for therapy-resistant hypertension, which is a frequently observed problem in the antihypertensive therapy. In previous studies, we used continuous sVNS to lower blood pressure (BP) without major side effects in a rat model. As continuous stimulation is energy consuming and sVNS could be implemented in an antihypertensive stimulator, it was the aim of this study to investigate the efficacy of
\end{abstract}

Enhanced content To view enhanced content for this article go to http://www.medengine.com/Redeem/ C3D4F0602924B2AB.

D. T. T. Plachta · J. Zentner · M. Gierthmuehlen ( $₫)$ Department of Neurosurgery, Medical Center, Faculty of Medicine, University of Freiburg, Breisacher Str. 64, 79106 Freiburg, Germany e-mail: mortimer.gierthmuehlen@uniklinik-freiburg.de

\section{Aguirre · T. Stieglitz}

Laboratory for Biomedical Microtechnology,

Department of Microsystems Engineering-IMTEK,

University of Freiburg, Georges-Koehler-Allee 106,

79110 Freiburg, Germany

O. Cota

Neuroloop GmbH, Engesserstr. 4, 79108 Freiburg, Germany pulsatile, cardiac-cycle-synchronized sVNS (cssVNS) on the reduction of BP.

Methods: A multichannel cuff electrode was wrapped around the left vagal nerve in six male Wistar rats under Isoflurane anesthesia. BP was recorded in the left carotid artery. An electrocardiogram (ECG) was obtained via subcutaneous needle electrodes. The aortic depressor nerve fibers in the vagal nerve bundle were selectively stimulated with 18 parameter settings within a window of 15-30 ms after the R-peak in the ECG. The stimulation paradigm included every heartbeat, every second heart beat, and every third heart beat. BP and heart rate were initially recorded over $10 \mathrm{~min}$.

Results: Using cssVNS, BP could be significantly reduced over $30 \mathrm{~min}$ and maintained at this level. While the highest BP reduction was seen during cssVNS at every heartbeat with minimal bradycardia, less-yet significant-BP reduction was seen during cssVNS at every second or third heartbeat without causing detectable bradycardia.

Conclusion: cssVNS can chronically reduce BP in rats avoiding measurable bradycardic side effects. This energy-efficient technique might 
allow the implementation of sVNS using an implantable device to permanently lower BP in patients.

Funding: The study was funded by Bundesministerium fur Bildung und Forschung/German Federal Ministry of Education and Research among the call "Individualisierte Medizintechnik" under the grant number FKZ 13GW0120B.

Keywords: Baroreflex; Hypertension; Neuromodulation; Pulsatile; Selective vagal nerve stimulation; Static

\section{INTRODUCTION}

Arterial hypertension is a common disease affecting millions of patients worldwide. Despite the development of new efficient medicine, up to $50 \%$ of hypertensive patients do not achieve a systolic target blood pressure (BP) below $140 \mathrm{mmHg}$ even under treatment with three antihypertensive drugs [1]. Non-adherence to medical therapy can be one cause, but it is believed that $5-13 \%$ of all those patients are truly therapy resistant [2]. Non-medical, device-mediated therapies are being investigated to address this problem [3, 4]. Renal denervation was successful in reducing BP in the Symplicity I (NCT00664638) and II (NCT00888433) studies, but failed to beat the control-group in Symplicity III (NCT01418261) [5]. While its complication rate was high in the first Rheos-trial (NCT00442286), carotid sinus stimulation still lowered the BP by $30 \mathrm{mmHg}$ over several years $[6,7]$.

Baroreceptors residing in the aortic arch transduce the arterial pressure curve into a neural signal, which is transferred to the nucleus of the solitary tract in the brainstem via the aortic depressor nerve ( $\mathrm{ADN})$. In rats, the
ADN is a separate fascicle, typically on the surface of the vagal nerve. Once the $\mathrm{ADN}$ is surgically freed from its surrounding tissue and detached from the vagal nerve, bipolar electrical stimulation can activate the baroreflex and lower BP by reducing the sympathetic tone [8]. As surgical dissection is not an option in human applications, we seek a technique for selectively stimulating the ADN fibers within the vagal nerve bundle by simply wrapping a multichannel cuff electrode (MCE) around the vagus and the accompanying tissue. Once the ADN fibers are detected using a coherent averaging algorithm, we are able to selectively reduce BP without provoking marked bradycardia, which would have been caused by the simple bipolar stimulation of the vagal nerve [9]. In this previous study, we used the static, continuous selective vagal nerve stimulation (sVNS) to immediately reduce BP by up to $60 \%$. As such stimulation might be too energy consuming for the permanent use in a potential neural stimulator, we sought to investigate whether pulsatile, pulse-synchronized sVNS might be an efficient alternative in lowering the BP.

Previous studies have demonstrated that the pulsatile mechanical stimulation of the baroreceptors is more efficient than static pressure in reducing BP $[10,11]$. This idea was supported by the observation that the physiologic activity of the ADN is also pulsatile instead of static due to the nature of the pulse wave; i.e., the firing pattern is phasic. Mimicking and overwriting this physiologic signal might potentially be more efficient, as the brainstem expects such pulsatile firing rather than constant high ADN activity. We, therefore, wanted to investigate whether sVNS synchronized with the cardiac-cycle-synchronized (cssVNS) could also reduce $\mathrm{BP}$ in a Wistar rat model. 


\section{METHODS}

The stimulation setup and signal analysis have been previously described $[9,12]$.

\section{Surgery}

We anesthetized all six male Wistar rats with 2-4 vol.\% Isoflurane (Abbott, North Chicago, Illinois, USA) and applied carprofen for analgesia (Rimadyl $^{\circledR}$, Pfizer, New York City, USA, $5 \mathrm{mg} / \mathrm{kg}$ bodyweight (BW) subcutaneously). We maintained constant anesthesia with 1-2 vol.\% Isoflurane and regulated the dose according to the respiration rate (RR). Isothermia was maintained with an isolated regulated heat pad (Harvard Apparatus, Holliston, Massachusetts, USA), and blood volume was kept constant with saline solution through an intravenous tail catheter ( $1 \mathrm{ml} / 100 \mathrm{~g}$ BW per $\mathrm{h})$. The left neurovascular sheath containing the vagal nerve, ADN, connective tissue, and some small blood vessels was approached through a ventral neck midline incision. Under a neurosurgical microscope (VM900, Moeller-Wedel GmbH, Wedel, Germany), the left carotid artery was ligated and cut cranially, and an aneurysm clip was used to achieve temporal occlusion of the caudal carotid artery for the placement of an intra-arterial microtip transducer (1.5 French, Millar Instruments, Houston, Texas, USA). The transducer was placed at the Waynforth position [13] and secured with ligatures. The MCE was connected to the data acquisition system and wrapped around the vagal nerve bundle, while the microtip transducer was attached to an in-house-designed amplifier. The amplified BP signal was transferred to the analog input of the data acquisition system. Both the electrocardiogram (ECG) and the transducer signal were digitized with identical parameters using separate input channels of the setup.
MCE

Flexible MCEs were manufactured as previously described [14]. Our MCE exhibited 24 contacts, which were equally pitched in eight tripoles around the cuff perimeter with a $45^{\circ}$ spacing [15]. Neurograms were recorded in a true-tripolar manner by the three electrode rings in the center of the cuff cylinder. The two electrodes placed outside of the cuff served as references. The MCE was 10-mm long and had an inner diameter of $0.8 \mathrm{~mm}$. The longitudinal distance between the contacts was $2 \mathrm{~mm}$. The contacts and interconnection lines were manufactured between two layers of polyimide with a total thickness of $11 \mu \mathrm{m}$. The 300-nm sputtered platinum thin-film metallization was coated with $1000 \mathrm{~nm}$ of sputtered iridium oxide on the contact sites. The ECG was recorded with three subcutaneous needle electrodes on the right and left chest and the left foot.

\section{Identification of Baroreceptor Fibers}

We used a PZ3 system (Tucker Davis Technology [TDT], Florida, USA) coupled with an RZ2 module linked to a Windows computer. This TDT system provided dynamic amplification of up to $60 \mathrm{~dB}$, automatically adapting the gain of the signal strength and matching it to the voltage range (here $\pm 3 \mathrm{mV}$ ). The signals from all contacts were subjected to monopolar amplification at a sampling rate of $12 \mathrm{kHz}$. A Matlab script (Version, 2015a, Mathworks, Natick, Massachusetts, USA) provided a 50-Hz notch- and a band-pass filter (Butterworth Second-order filter, 20-200 Hz). All experiments were performed in a copper faraday cage connected to a common ground with all other experimental devices. The monopolar recordings were combined with tripolar recordings through two-stage 
amplification, as described in previous work [9]. The tripole contacts located near the baroreceptor fibers (ADN) were identified through coherent averaging [16]. These contacts were chosen for the stimulation procedure described below.

\section{Static and Pulsatile sVNS}

All stimulation consisted of a given number (one sweep consists of $\leq 100$ pulses) of current-controlled, charge-balanced biphasic rectangular pulses. The pulses were generated in the PZ3 system (16-bit resolution, $24 \mathrm{kHz}$ conversion rate) and transferred to an in-house-constructed eight-channel voltage-to-current converter. For the stimulation, the two peripheral ring electrodes of the MCE were set as anodes, against one of the center electrodes of the recording tripole near the baroreceptor fibers, which was chosen as the cathode. The identified stimulation sites were further verified through manually controlled test stimulation. These test stimulation were initiated at a very low-amplitude $\quad(<0.2 \mathrm{~mA})$ and became increased in a stepwise manner to track the responsiveness of the stimulation site. The test stimulations were used to find the threshold for the beginning of a BP-decreasing response and the threshold for critical stimulation (BP decrease $>50 \%$ ). The automatized stimulation pattern was subsequently set between these amplitude thresholds.

\section{Experimental Procedure, Data Analysis, and Statistics}

After surgery and prior to any stimulation, recordings were performed in each animal for 10 min to obtain the basic vital parameters and to allocate sufficient data for localization of the appropriate stimulation site (see Fig. 1). Then, test stimulations were applied to the identified recording sites to obtain the threshold criteria. Following the test stimulation sequence, the animals were allowed recover for 5-10 min.

This recovery phase was followed by a sequence of automatized static stimulations. The stimulation parameters were the repetition rate $(30,40$ and $50 \mathrm{~Hz})$, amplitude (0.2-0.9 $\mathrm{mA})$ and pulse width $(0.2-0.9 \mathrm{~ms})$. All static stimulation sweeps, included 100 pulses, and were repeated three times. Within a sweep, stimulation parameters were not changed. The repetition rate of the stimulation altered the stimulation duration. Every stimulation sweep (100 pulses) with a repetition rate of $30 \mathrm{~Hz}$ had a total duration of $3.3 \mathrm{~s}$, while each $40 \mathrm{~Hz}$ sweep had duration of $2.5 \mathrm{~s}$, and each $50 \mathrm{~Hz}$ sweep had duration of $2 \mathrm{~s}$. The test animals exposed individual thresholds to the stimulation amplitude. We adapted the stimulus parameter settings according to the responsiveness of the individual test animal and did not obtain the same lowest and highest values (amplitude and pulse width) across all test species. We combined all three repetition rates with at least five different amplitudes and at least five different pulse widths. Each set of stimulation parameters was repeated three times in the static, continuous phase. The parameter settings were applied in an increasing fashion. Between each sweep, there was a pause (inter-sweep-interval) of at least $10 \mathrm{~s}$. After three sweeps, a pause of $25 \mathrm{~s}$ was embedded.

The static stimulation phase was followed by another 5-min recovery phase. The pulsatile stimulation sequence was initiated using the same settings for the stimulation parameters. For the pulsatile stimulation, each stimulus sweep of 100 pulses had to be collapsed to a series of pulses that would fit the refractory phase of the heartbeat, i.e., the time of blood 

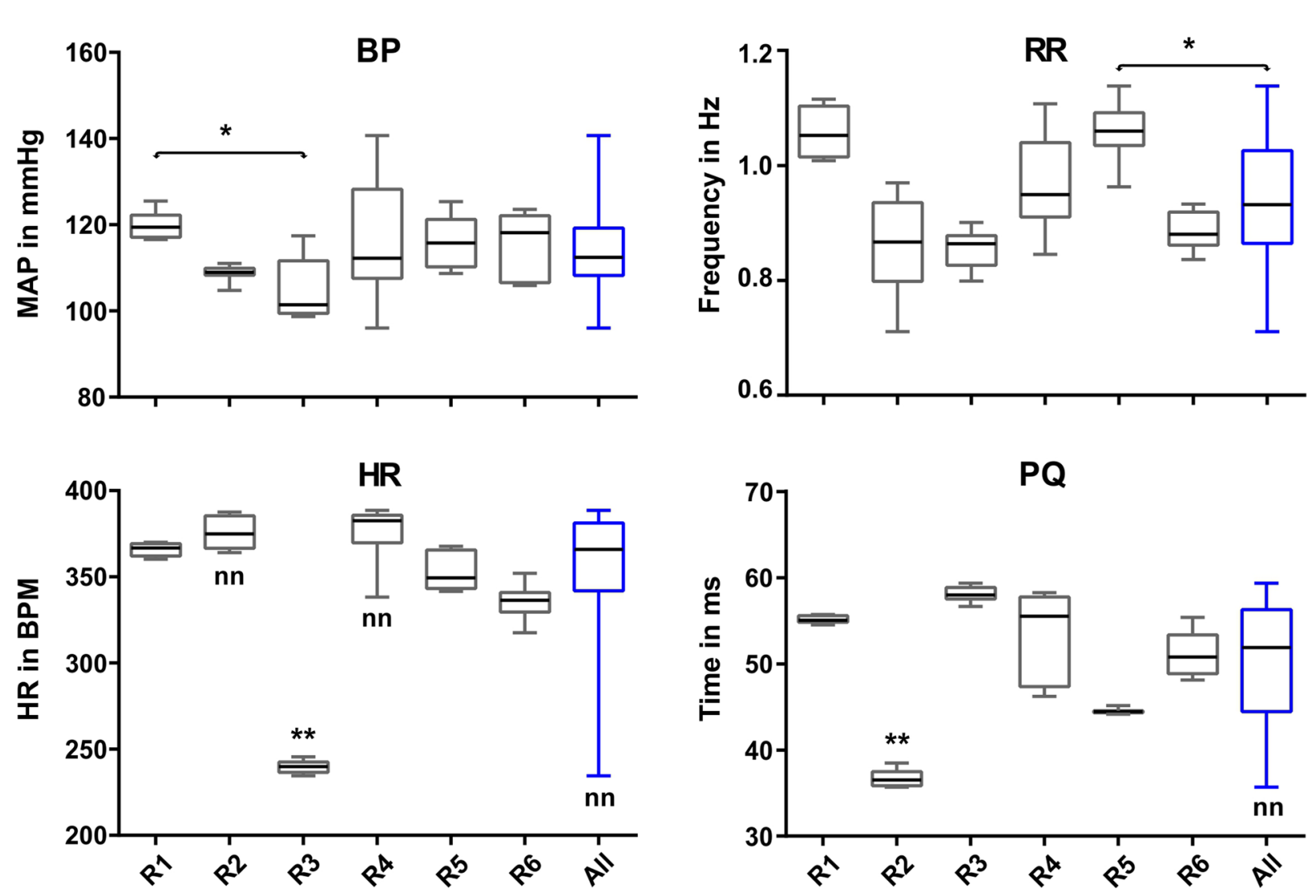

Fig. 1 Initial readings of all animals. Blood pressure (BP), respiration rate $(R R)$, heart rate $(H R)$, and PQ-time (PQ) of all six test animals (rat $1-6=\mathrm{R} 1-\mathrm{R} 6$ ) after surgery, prior to stimulation. The boxplots represent the median with the upper and lower quartile. All individually pooled vital data were tested for normality. Data sets that failed these tests were labeled not-normal (nn) for non-normal distributed. The blue boxplot in each subplot features the pooled data of all six test animals. All animals showed average BP [in mean arterial pressure (MAP)] between 100 and $120 \mathrm{mmHg}$. The test animals possessed a RR between 0.8 and $1.2 \mathrm{~Hz}$ with test animal one remaining completely

expulsion. The number of pulses in this time window is a function of the repetition rate of the stimulus. For $30 \mathrm{~Hz}$, we used four pulses; for $40 \mathrm{~Hz}$, we used five pulses; and for $50 \mathrm{~Hz}$, we used seven pulses (see Fig. 2). To get as close as possible to the total of 100 pulses per sweep in the static stimulation, we repeated the pulsatile stimulation for 25 heart beats for $30 \mathrm{~Hz}$, for 20 heart beats for $40 \mathrm{~Hz}$, and for 14 heart beats for

beyond $1 \mathrm{~Hz}$. The $\mathrm{HR}$ of five animals was in the range of 340-380 beats per minute (BPM). Animal three showed a rather low, but sustained HR in the control measurement resulting in a long extension of the pooled boxplot. The PQ-times of five animals ranged between 45 and $58 \mathrm{~ms}$. PQ of animal two was rather low at $38 \mathrm{~ms}$. If a sample showed a significant difference to any other sample, this was indicated by a star and a linked line. If a sample featured a significant difference to all other samples, the maximum number of stars (highest $P$-level) was placed above this sample. ns $P>0.05 ;{ }^{*} P \leq 0.05 ;{ }^{* *} P \leq 0.01$; ${ }^{* * *} P \leq 0.001 ;{ }^{* * * *} P \leq 0.0001$

$50 \mathrm{~Hz}$. Because pulsatile stimulation was triggered by the ECG artifact, the total duration of each stimulation setting depended on the heart rate (HR) during the stimulation (see Fig. 2). Each setting of the stimulation parameters was repeated five times. The inter-sweep interval was always $10 \mathrm{~s}$. In the first pulsatile recording, we used every heartbeat to trigger stimulation (P1). After this 


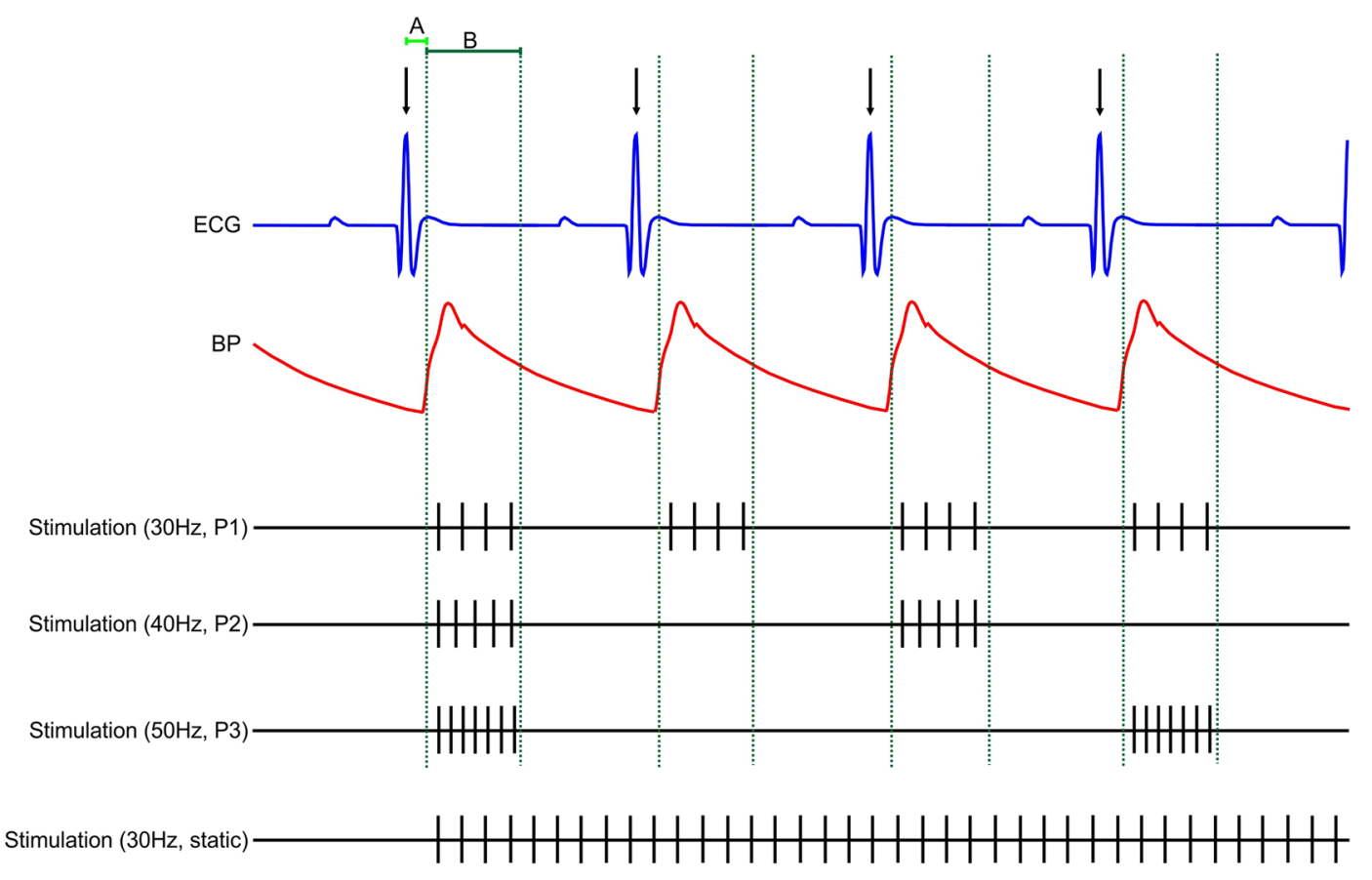

Fig. 2 Schematic on composition of static vs. pulsatile stimulation. Top trace (blue) indicates the ongoing electrocardiogram (ECG) signal. The red trace underneath features the according blood pressure recording (from the tip catheter in the aortic arch). Each R-peak of the ECG signal was detected by the software (black arrow indicates the detection). From each R-peak, a given (fixed) delay was obtained ( $a$, light green). In addition, a second interval ( $b$, dark green) was obtained, starting right after the first delay and ending half way prior to the next ECG. Within this green interval, the stimulation pulses of the pulsatile stimulation were placed (indicated by the dotted green

stimulation sequence, the test animal was allowed another $5 \mathrm{~min}$ to recover. This was followed by a pulsatile stimulation sequence in which we used the same settings, except that we now employed only every second heart beat for stimulation (P2). This was followed by another recovery phase and by a third sequence of pulsatile stimulation, in which we used only every third heart beat for stimulation (P3). Because the interval between the R-peak of the ECG and the increase in the BP pulse wave did
Time $900 \mathrm{~ms}$

vertical lines). The four traces below the red blood pressure (BP) trace illustrate with three examples how the different stimulation settings were arranged (from top to bottom): $30-\mathrm{Hz}$ pulsatile stimulation at every heartbeat (P1), $40 \mathrm{~Hz}$ Stimulation at every second heartbeat (P2), $50 \mathrm{~Hz}$ stimulation at every third heartbeat (P3) and continuous (static) stimulation. All stimulations consisted of 100 pulses (only $50 \mathrm{~Hz}$ was, by calculation, 98 pulses) and were applied over different times: while at $50 \mathrm{~Hz}$, these pulses were applied over a shorter time ( $2 \mathrm{~s})$, it took $3.3 \mathrm{~s}$ during $30 \mathrm{~Hz}$. At the end, the same amount of energy was applied to the nerve, making all results comparable

not change during the stimulation, this time interval was individually set at the beginning of each pulsatile stimulation sequence using the in-house-written software in Matlab 2015a.

\section{Data Analysis and Statistics}

During the initial recording of the vital parameters, all data were pooled and analyzed to determine whether they showed a normal distribution using the D'Agostino-Pearson 
omnibus, Kolmogorov-Smirnov, and Shapiro-Wilk tests for normality.

During the baseline recording, we extracted mean arterial pressure (MAP), HR, and RR from the $\mathrm{BP}$ recordings from the tip catheter, and the PQ interval from the ECG signal using an in-house-written Matlab program. Because the stimulation disturbed the ECG recording, we could only record BP, HR, and the RR during stimulation. For each stimulation sweep, the initial BP was obtained in a 2-s measurement prior to the initiation of the stimulation. The decrease in BP as a result of each stimulation was determined from the delta of MAP between the initial BP prior to the beginning of the stimulation against the trough of the MAP curve obtained during the stimulation sweep and an 8-s interval afterwards. Changes in HR during the stimulation were obtained from the cycles in the MAP data and compared with the HR prior to pulsatile or static stimulation. Respiration was not calculated as a rate, because the number of cycles during stimulation was insufficient. However, we used respiration to detect whether apnea occurred during or after the stimulation sweeps in both scenarios (static and pulsatile stimulation).

The BP and HR data for each stimulation sweep of both static and pulsatile stimulation were pooled over all six animals. The pooled data were analyzed for a normal distribution using the Kolmogorov-Smirnov or the Spiro-Wilks test. For the sequential pairwise testing, either ANOVA or the non-parametric sequential Kruskal-Wallis test was used. To achieve a better cross-compatibility, the decreases in BP and HR were also normalized as percentages against the initial values.

Apnea, defined as an unexpected pause between two regular respirations during stimulation, could only occur once or twice during stimulation. Apnea was, therefore, calculated from its occurrence over the pooled trials for all six animals. To measure the efficacy of the stimulation, the ratio of the decrease in BP (in mmHg) over the decrease in HR [in beats per minute (BPM)] was calculated and visualized in a semi-logarithmic plot using Matlab and Prism 6.0 (Graphpad, La Jolla, California, USA).

\section{Compliance with Ethics Guidelines}

This study was approved by the Regierungspraesidium Freiburg, Baden-Wuerttemberg, and the Ethics Committee of the University of Freiburg (G13-44). All institutional and national guidelines for the care and use of laboratory animals were followed.

\section{RESULTS}

\section{Baseline Vital Parameters}

The overall average MAP was $114 \mathrm{mmHg}$ $( \pm 9.2 \mathrm{mmHg}$ STD, median $=113 \mathrm{mmHg})$ (see upper left blue box in Fig. 1). The MAP data for all individual test animals as well as the overall pooled data were normally distributed. In a sequential ANOVA test (Holm-Sidak's multiple comparisons), we observed a significant difference only between the BP of rat 1 vs. rat $3(P=0.05)$ (see Fig. 1BP). The overall average RR was found at $0.94 \mathrm{~Hz} \quad( \pm 0.1 \mathrm{~Hz} \quad S T D$, median $=0.93 \mathrm{~Hz}$ ). Although the six animals showed a wide range in RRs, all individual and pooled distributions passed the tests for normality. Through parametric multiple comparisons, we found several significant differences between the individual RRs of individual rats. In particular, the RR of rat 5 showed a significant difference against the pooled RR data. Despite the outlier quality of this result, it is still within the range of normal 
RRs in anesthetized rats and was kept in the pooled distribution.

The average HR was $350 \mathrm{BPM}$ ( \pm 45 BPM STD, median =366 BPM). Because some samples failed the test of normality (HRs marked "nn" in Fig. 1), we used a non-parametric multiple test (Kruskal-Wallis with Dunn's multiple test) to determine which samples were significantly different. The HR of rat 3 was significantly lower than the sampled HRs of all other test animals and was also significantly lower compared with the pooled sample $(P<0.01$, Dunn's multiple test). For the sake of completeness and because even an average HR of 240 BPM is still considered normal, we kept this sample in the overall pool.

The average PQ interval occurred at $50 \mathrm{~ms}$ ( $\pm 7 \mathrm{~ms}$ STD, median $=51 \mathrm{~ms})$. Again, we observed at least one animal exhibiting a significant deviation from the samples of all other test species. In rat 2, the PQ interval (average $=36 \mathrm{~ms}, \pm 1 \mathrm{~ms}$ STD) was found to be significantly lower than in all other animals $(P<0.01$, Dunn's multiple test). Although the data for this sample caused the pooled sample to fail the test of normality, we retained it in the analysis for the same reasons, as in the previous cases.

Across all four vital parameters, we did not find an individual test animal that exhibited significant differences in all four parameters against the pooled sample. In rats 2, 3, and 5, we observed individual "glitches" in one of the four vital parameters. The reason for these individual differences was not identified.

\section{Static, Continuous Stimulation}

\section{Decrease in BP}

Static stimulation leads to a graduated BP decrease depending on the settings of the stimulation. Figure 3a shows the responses of
BP, MAP, HR, and RR to one stimulation sweep $(30 \mathrm{~Hz}, 0.5 \mathrm{~ms}, 0.5 \mathrm{~mA})$ in one animal. Even with sVNS, some bradycardia could not be avoided. The violin plot shown in Fig. 4 illustrates the pooled responses of all six test animals regarding BP (left column) and HR (right column). In comparison with the repetition rate, the greatest average reduction in MAP was observed at $40 \mathrm{~Hz}$ (average $=28 \mathrm{mmHg}, \pm 20 \mathrm{mmHg} \mathrm{STD}$, median $=24 \mathrm{mmHg})$. At $30 \mathrm{~Hz}(25 \mathrm{mmHg}$, $\pm 20 \mathrm{mmHg}, 20 \mathrm{mmHg})$ and $50 \mathrm{~Hz}(13 \mathrm{mmHg}$, $\pm 13 \mathrm{mmHg}, 12 \mathrm{mmHg}$ ), there was a smaller decrease in BP. At $50 \mathrm{~Hz}$, the pooled MAP decrease was even significantly smaller compared with the pooled results obtained at $40 \mathrm{~Hz}$ (Dunn's multiple test, $P<0.05$ ).

To achieve better comparisons, the amplitude results were pooled into three classes: low-amplitude stimulation (ranging from 0.2 to $0.4 \mathrm{~mA}$ ), mid-amplitude stimulation (ranging from 0.5 to $0.6 \mathrm{~mA}$ ), and high-amplitude stimulation (ranging from 0.7 to $0.9 \mathrm{~mA}$ ). The strength of the decrease in MAP increased almost linearly with an increasing stimulation amplitude (low-amp: 16, \pm 18 , $12 \mathrm{mmHg}$ ), (mid-amp: 26, $\pm 17,23 \mathrm{mmHg}$ ), (high-amp: 30, $\pm 17,33 \mathrm{mmHg}$ ). Sequential comparisons (Dunn's multiple test) revealed significant differences between all three amplitude levels, except for the mid- vs. high-amplitude comparison $(P<0.01)$.

Similar to the amplitude data, the pulse width results were pooled into three different classes: short (ranging from 0.2 to $0.3 \mathrm{~ms}$ ), intermediate (ranging from 0.4 to $0.5 \mathrm{~ms}$ ), and long (ranging from 0.6 to $0.9 \mathrm{~ms}$ ). In contrast to the findings for the amplitude, the pulse width did not scale up the decrease in BP with an increasing duration. The peak occurred in the mid-range pulse width $(27, \pm 22,24 \mathrm{mmHg})$. Shorter $(21, \pm 17,17 \mathrm{mmHg})$ and longer (16, $\pm 12,13 \mathrm{mmHg}$ ) pulse widths were less effective. 
(A) Static Stimulation
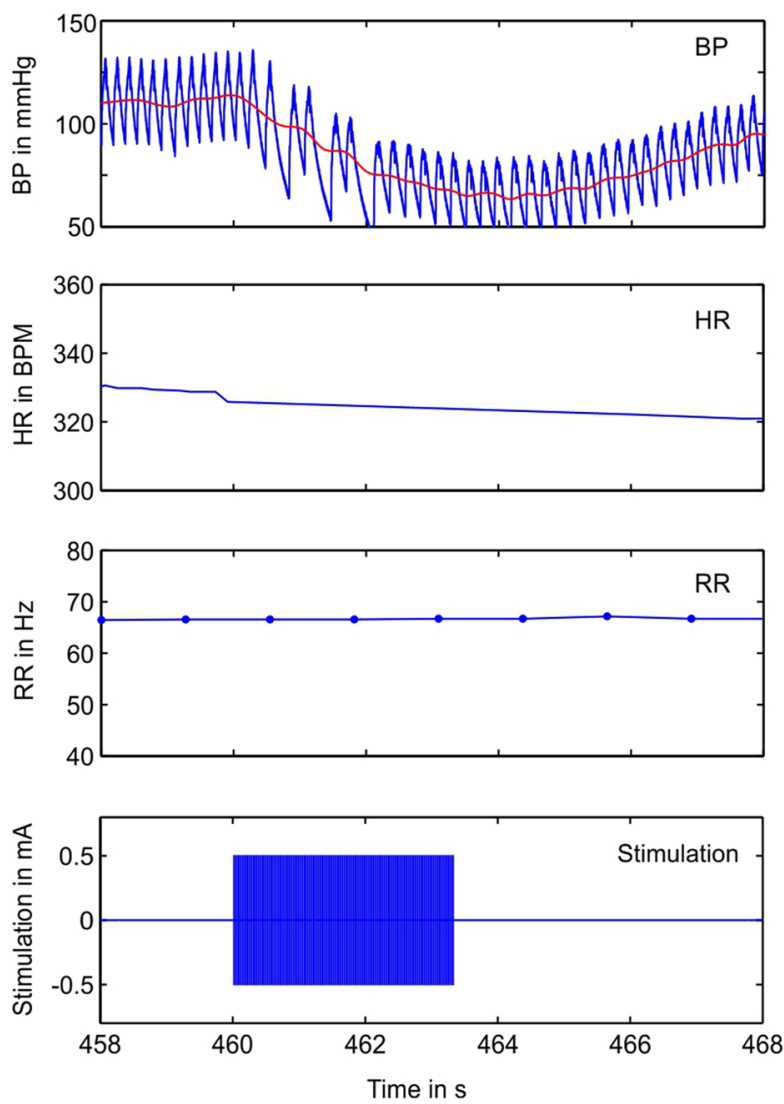

Fig. 3 Example of continuous (a) and pulsatile (b) selective vagal nerve stimulation. a Example response during one sweep of a continuous stimulation at $30-\mathrm{Hz}$ repetition rate and $0.5-\mathrm{mA}$ amplitude (highlighted by the red frame). The first column shows (top down) the blood pressure (BP) in $\mathrm{mmHg}$, the heart rate (HR) in beats per minute (BPM), and the respiration rate $(\mathrm{RR})$ in $\mathrm{Hz}$ as a response to the stimulation illustrated by the stimulation track at the bottom. Note that the RR was integrated and integration points are shown in the according subplot. This example was chosen, as it features mild, but visible side effects on the HR (see especially the top trace). b Example from the same

However, the differences between the three groups did not reach the level of significance.

\section{Decrease in HR}

HR was more strongly affected by lower stimulation frequencies. At $30 \mathrm{~Hz}$, bradycardia was greater $(44, \pm 33,40 \mathrm{BPM})$ compared with $40 \mathrm{~Hz}(35, \pm 28,30 \mathrm{BPM})$ and $50 \mathrm{~Hz}(27, \pm 22$,
(B) Pulsatile Stimulation (P1)
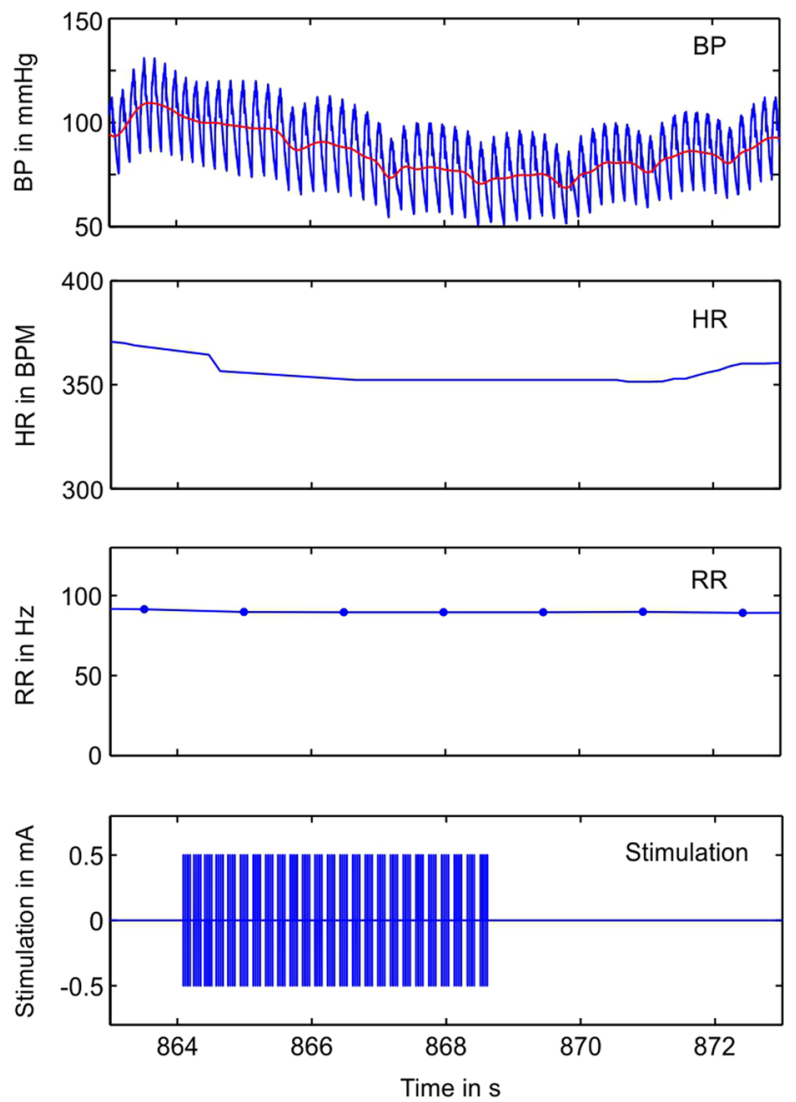

test animal with the same stimulation parameters, this time illustrating the response to one sweep of pulsatile stimulation at $30 \mathrm{~Hz}$ and $0.5-\mathrm{mA}$ amplitude. The arrangement of this figure is analogous to Fig. 2 with the exception that the time line is longer, since the pulsatile stimulation requires more time for 100 pulses. The blue stimulation "packs" correspond to the four pulses of the "stimulation $30 \mathrm{~Hz} \mathrm{P1"} \mathrm{trace} \mathrm{of} \mathrm{Fig.} \mathrm{2.} \mathrm{While} \mathrm{the} \mathrm{BP}$ reduction appears rather free from side effects, the inter stimulus intervals (corresponds with the HR) slightly extend at the beginning of the stimulation

$25 \mathrm{BPM})$. It is noteworthy that an even a reduction of $44 \mathrm{BPMs}$ is equivalent to bradycardia slightly above 10\%. However, these differences remained below the level of significance.

As for BP, bradycardia was driven by an increasing stimulation strength. From low-amp (26, $\pm 24,26 \mathrm{BPM})$ to mid-amp $(41, \pm 30$, 

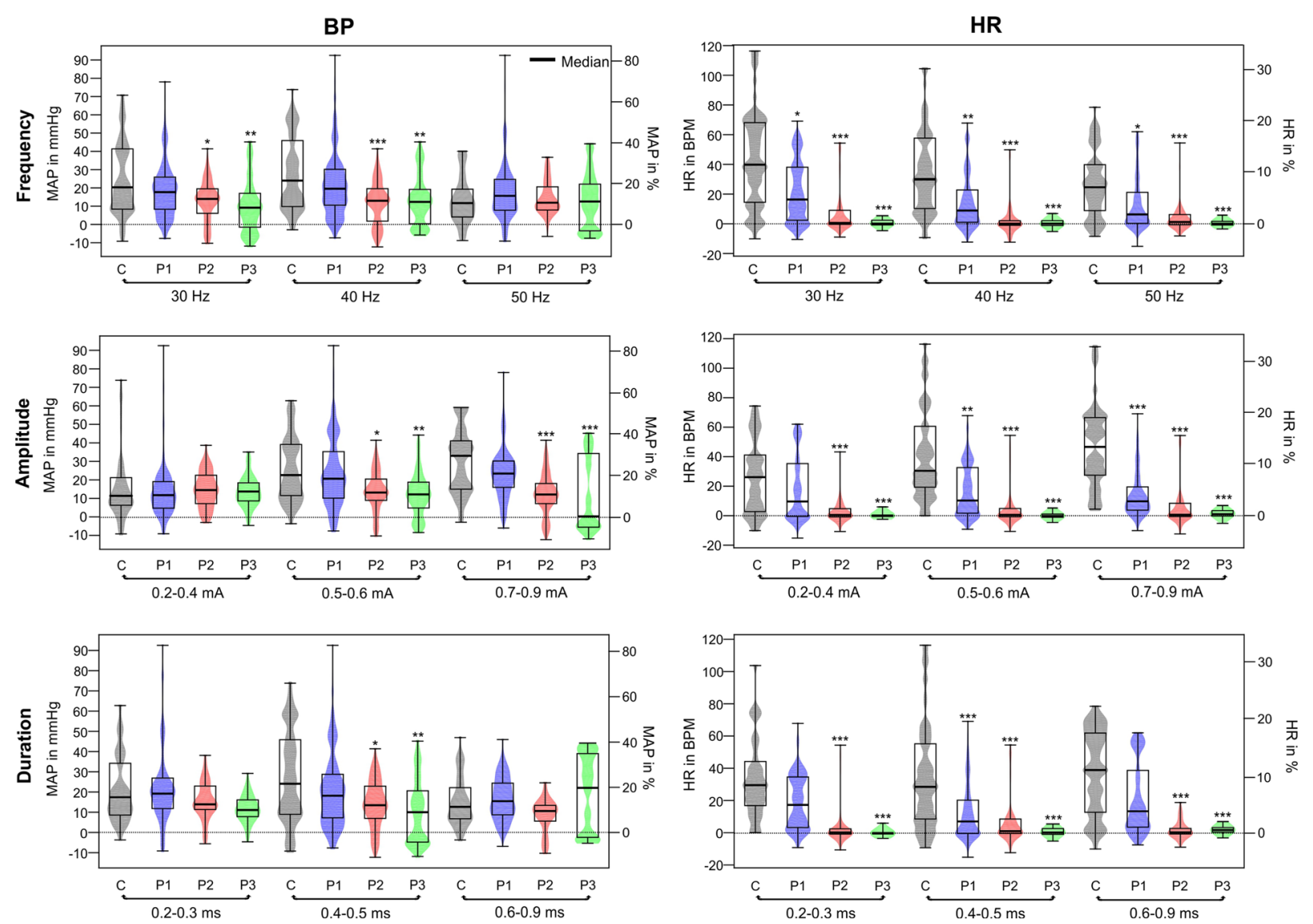

Fig. 4 Analysis of heart rate and blood pressure during selective vagal nerve stimulation. Blood pressure (BP) decrease (first column) and heart rate (HR) decrease (second column) as a function of stimulation frequency (first row), stimulation amplitude (second row), and stimulation duration (third row). The violin plot underneath the boxplots illustrates the distribution function of the data sample, resulting in the boxplot. Gray plots present control (constant stimulation), blue (P1), red (P2), and green (P3) feature pulsatile stimulation at every heartbeat (P1), every second (P2), and every third (P3). All plots feature two scale bars, the left shows the true measure, and the right the

normalized measure (in \%). In each plot, the control was tested against the pulsatile results for significant differences. Significant differences were marked with an asterisk. While the pulsatile stimulation shows similar capabilities to reduce the BP (at least P1 and P2), the pulsatile stimulation is generally outperforming the constant stimulation in terms of bradycardia. Especially in $\mathrm{P} 2$ and $\mathrm{P} 3$, the average $\mathrm{HR}$ often featured a stimulus-related bradycardia below the threshold of detection (<4\%). MAP, mean arterial pressure. ns $P>0.05 ; \quad{ }^{*} P \leq 0.05 ; \quad{ }^{* *} P \leq 0.01 ; \quad{ }^{* * *} P \leq 0.001$; ${ }^{* * * *} P \leq 0.0001$

$31 \mathrm{BPM})$ and high-amp $(48, \pm 29,47 \mathrm{BPM})$, bradycardia continued to increase. Another effect became obvious (especially in the HR responses) within the distribution within one group. The results showed strong spreading with a little homogeneity within the group (illustrating this pattern was the motivation for using the violin plot underneath the box plot).

The inter-individual differences in the responses to stimulation were the strongest in this regard. In pairwise comparisons, only the results from the low-amp stimulation showed a significant difference against the high-amp results (Dunn's multiple test, $P<0.05$ ).

The reduction of HR increased slightly with an increasing pulse width. From a short pulse 
width $(33, \pm 25,30 \mathrm{BPM})$ to an intermediate pulse width (34, $\pm 33,30 \mathrm{BPM})$, the increase was small, and the high pulse width had a stronger impact $(37, \pm 25,38 \mathrm{BPM})$. Again large inter-individual differences became obvious in the violin plot. However, the results for short, intermediate, and long pulse widths did not reach the level of significance using the Dunn's multiple test.

\section{Occurrence of Apnea}

The occurrence of apnea across the different stimulation parameters did not follow a linear pathway (see Fig. 5). Across all stimulation parameters, the greatest respirational side effects were found at the mid-level. This was the case for the frequency $(40 \mathrm{~Hz})$, stimulation amplitude (mid-strength), and pulse width (mid-duration). The lowest levels of apnea in the static stimulation were observed at a stimulation frequency of $30 \mathrm{~Hz}$.

\section{Pulsatile Stimulation}

\section{Decrease in $\mathrm{BP}$}

Pulsatile stimulation decreased BP and HR and, in some instances, also caused to apnea. The focus of this manuscript is the effectiveness of pulsatile vs. static stimulation, with the absolute stimulation energy as a constant (in terms of pulses). Figure 4 illustrates this comparison, including the effects of skipped heart beats during the pulsatile stimulation. The results of the static stimulation are shown in gray, while the results of the pulsatile stimulation are shown in blue (stimulation at every heartbeat $=\mathrm{P} 1$ ), red (stimulation at every second heartbeat $=\mathrm{P} 2$ ), and green (stimulation at every third heartbeat $=\mathrm{P} 3$ ).

With respect to the BP-decreasing power of pulsatile stimulation, the results of pulsatile stimulation at every heartbeat resemble the results of the static stimulation, with a slightly lower impact being observed at 30 and $40 \mathrm{~Hz}$ and a slightly higher impact at $50 \mathrm{~Hz}$. This difference between static stimulation and P1 did not become significant at any level. In all the cases, the skipping of heartbeats led to a reduction of the BP-decreasing power of the pulsatile stimulation. However, this was observed only in the pairwise comparisons of P2 and P3 against P1. The difference between the pulsatile stimulation applied in P2 and P3 was only measurable at $30 \mathrm{~Hz}$, with a small margin in favor of P2. Pairwise comparisons of each pulsatile stimulation treatment with the

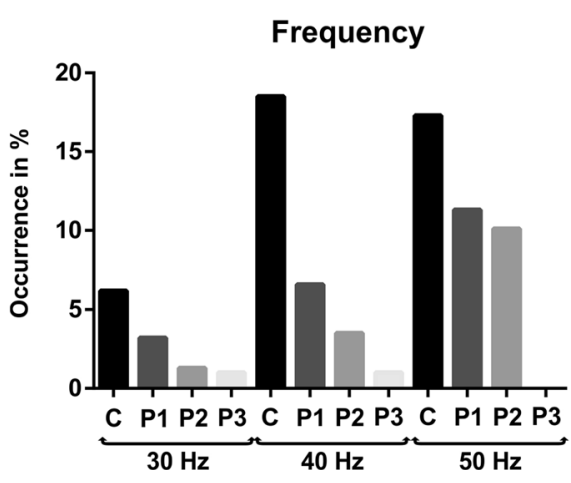

Fig. 5 Occurrence of apnea during selective vagal nerve stimulation. Apnea occurrence during stimulation as a function of stimulation frequency, stimulation amplitude, and stimulation pulse width. Note that under all but one
Amplitude

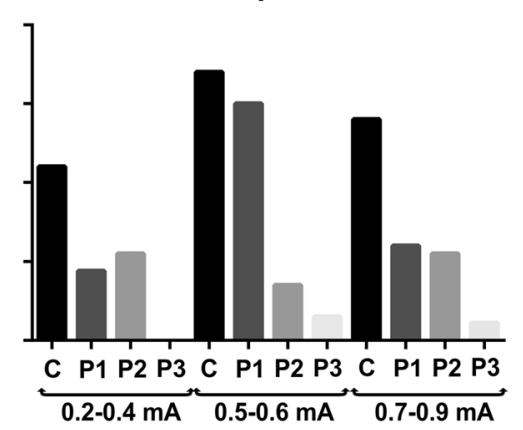

condition (P1, pulse width $0.2-0.3 \mathrm{~ms}$ ), the pulsatile stimulation featured lower apnea rates than the constant stimulation 
control (static stimulation) revealed a significant decrease in comparison with static stimulation only for P2 and P3 at 30 and $40 \mathrm{~Hz}$ (Dunn's multiple test $p<0.01$ ).

During the static stimulation, the BP-decreasing effect increased with the stimulation amplitude. The same effects were observed for pulsatile stimulation as long as every heartbeat was stimulated (P1). No significant difference could be detected between static stimulation and P1. Skipping single heartbeats altered the effectiveness of pulsatile stimulation. While pulsatile stimulation at every second or third heartbeat was even stronger at low-amplitudes compared with the control (though not significantly), the effectiveness remained rather constant for the pulsatile stimulation applied in P2 and decreased greatly in P3, especially at high-amplitudes.

The pulse width did not affect the results of P1 and P2. The decrease in BP remained stable under short, intermediate, or long pulse widths. However, results of P3 became rather inhomogeneous at very long pulse widths, leading to an overall increase. Static control only showed a significantly higher impact on the decrease in BP compared with P2 and P3 pulsatile stimulation under the most effective intermediate pulse width.

Again, the violin plot revealed that the results of pulsatile stimulation were generally more homogeneous than the results of static stimulation, with one exception: P3 pulsatile stimulation became highly inhomogeneous at the highest amplitudes and greatest pulse widths.

\section{Decrease in $H R$}

While pulsatile stimulation was almost as effective as static stimulation in terms of decreasing BP, the advantages of pulsatile stimulation became apparent in relation to side effects, such as bradycardia. Even P1 pulsatile stimulation resulted in a significant decrease in bradycardia compared with the static control (Fig. 4), with an average HR reduction of $21 \mathrm{BPM}$ being observed at $30 \mathrm{~Hz}$ $( \pm 20$ BPM STD, median =16 BPM $), 14 \mathrm{BPM}$ at $40 \mathrm{~Hz}( \pm 17$ BPM STD, median =9 BPM $)$ and 13 $\mathrm{BPM}$ at $50 \mathrm{~Hz}( \pm 17 \mathrm{BPM}$ STD, median $=6 \mathrm{BPM})$ (Dunn's multiple test). The results of P1 pulsatile stimulation, therefore, resemble the results of static stimulation (decreasing bradycardia with an increasing frequency), but at a significantly lower level. P2 pulsatile stimulation resulted in average bradycardia just above noise level: $7 \mathrm{BPM}$ at $30 \mathrm{~Hz}( \pm 15$ BPM STD, median $=0.6 \mathrm{BPM}), 4 \mathrm{BPM}$ at $40 \mathrm{~Hz}$ $( \pm 12$ BPM STD, median $=0.09 \mathrm{BPM})$, and 4 $\mathrm{BPM}$ at $50 \mathrm{~Hz}( \pm 12 \mathrm{BPM}$ STD, median $=1 \mathrm{BPM})$. Finally, during stimulation in P3, bradycardia fell within the range of noise: $0.7 \mathrm{BPM}$ at $30 \mathrm{~Hz}$ $( \pm 2$ BPM STD, median $=0.1 \mathrm{BPM}), 0.5 \mathrm{BPM}$ at $40 \mathrm{~Hz}( \pm 2 \mathrm{BPM}$ STD, median $=0.08 \mathrm{BPM})$, and $0.5 \mathrm{BPM}$ at $50 \mathrm{~Hz}( \pm 2 \mathrm{BPM}$ STD, median $=0.05$ $\mathrm{BPM})$.

All of the results of pulsatile stimulation were significantly lower than the results of static stimulation, with the exception of pulsatile stimulation of every heartbeat at a low-amplitude.

The pulse width had a minor effect on bradycardia under the P1 pulsatile stimulation. In P2 and P3, bradycardia showed almost no correlation with pulse width and remained at noise levels.

\section{Occurrence of Apnea}

All of the pulsatile stimulation conditions resulted in a lower occurrence of apnea with one exception: at a low pulse width, the results of P1 were slightly greater than in the control. Apnea became a rather rare side effect in P3, and 
was triggered only under certain combinations of stimulation.

\section{Efficacy Calculation}

The BP-reducing effect of pulsatile stimulation is slightly, but not significantly, weaker than that of static stimulation. However, bradycardia is markedly increased during static stimulation. In Fig. 6, we emphasized the correlation between the decrease in BP and bradycardia in a normed manner. To determine the efficacy of the stimulation, the decrease in $\mathrm{BP}$ in $\mathrm{mmHg}$ was divided by the reduction of HR in BPM. Because HR can reach values near or even below zero (resulting in false high or even negative efficacy values), we set all HR values below 1-1. Under all conditions, pulsatile stimulation outperformed static stimulation. Across the various stimulation frequencies, pulse widths, and amplitudes, P2 and P3 were significantly more effective than static stimulation in most cases. Values of $10 \mathrm{mmHg} / \mathrm{BPM}$ and higher could be achieved, while the most efficient static stimulation resulted in a value of only $1 \mathrm{mmHg} / \mathrm{BPM}$ at the lowest stimulation amplitude with a 40-Hz stimulation frequency. The difference between P2 and P3 was typically small. In 10 out of 18 cases, P3 stimulation was less effective than P2 stimulation; however, the highest efficacy values were obtained in P3 at $50 \mathrm{~Hz}$ with the longest pulse width.

\section{DISCUSSION}

Although medical therapy is the first choice for treating arterial hypertension, up to 50\% of patients do not achieve a target BP of below $140 \mathrm{mmHg}$ [1]. Not every patient has therapy-resistant hypertension, and non-compliance can be one of the reasons for this potentially fatal situation [17], but between
$8 \%$ and $13 \%$ of all hypertensive patients are considered to be truly therapy resistant [18].

One device-mediated antihypertensive approach is activation of the baroreflex via CSS, which has been investigated since the 1960s by Bilgutay and Neistadt [19-21]. Modern CSS has been demonstrated to permanently lower BP by up to $30 \mathrm{mmHg}$ over several years, showing that the concept of antihypertensive baroreflex activation is sound; however, the periprocedural complication rate is high $[6,7]$.

Bipolar vagal nerve stimulation has been used to treat therapy-resistant epilepsy since the 1990s, and is associated with a low complication rate [22]. Based on the notion that there are baroreceptor fibers within the vagal nerve from the aortic baroreceptors that are also capable of activating the baroreflex, we recently proposed the technique of antihypertensive sVNS in rats [9]. We found that simultaneously administered Metoprolol or Enalapril only slightly reduces the efficacy of sVNS in rats, without altering its underlying mechanisms [12, 23].

In our initial study on sVNS, we were able to reduce BP by up to $60 \%$ through continuous stimulation over several seconds, but continuous static electrical stimulation is energy consuming and stressful for both materials and tissues. As we plan to implement sVNS in an implantable stimulator device for long-term antihypertensive therapy, we wanted to investigate if pulsatile, energy-efficient sVNS can also reduce BP. One of the important aspects of pulsatile stimulation is, that with the same amount of charge (counted as number of pulses), the BP reduction can reach similar values as during continuous stimulation, with far less side effects (HR and RR). Due to the pulsatile forming of stimulation "packs" of 4-7 pulses per heartbeat, the overall stimulation 

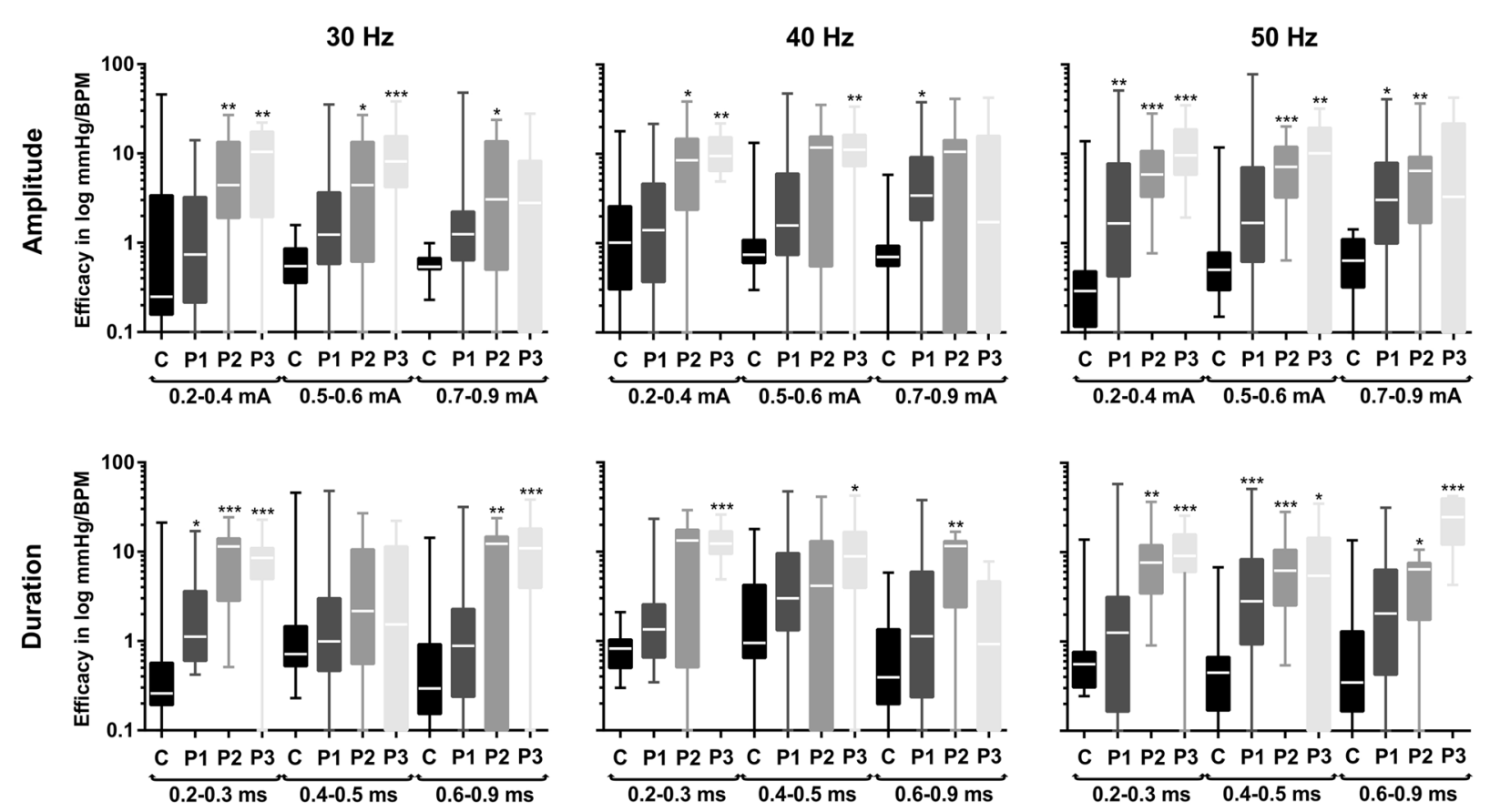

Fig. 6 Efficacy of selective vagal nerve stimulation in continuous and pulsatile modes. Semi-logarithmic stimulation efficacy plot over all three stimulation parameters: frequency (columns), amplitude (first row), and pulse width (second row). Significant differences were calculated against the control under each stimulation setting. While the best average

time for 100 pulses per sweep massively increased, even though the same amount of energy was used. In other words, pulsatile stimulation lowers side effects and lasts longer. The only possible downside of pulsatile vs. static stimulation is the fast and steep response of static stimulation on the BP. Ideally, an implant should be able to run in both "modes" using continuous sweeps to reduce BP fast and with a high delta and pulsatile stimulation to hold a certain level of BP.

The Nucleus tractus solitarii is the center for BP regulation of the body and receives inputs from both, the carotid sinus and the aortic baroreceptors. It consists of subgroups of neurons that react with different sensitivities to either static or pulsatile changes in BP. It is performance for constant stimulations was found at $40 \mathrm{~Hz}$ and $0.2-0.4 \mathrm{~mA}$ [with an efficiency of $1 \mathrm{log}(\mathrm{mmHg} / \mathrm{beats}$ per minute $[\mathrm{BPM}])]$, the corresponding values for pulsatile stimulation are always higher, and in most cases, highly significantly so. ns $P>0.05 ; \quad{ }^{*} P \leq 0.05 ; \quad{ }^{* *} P \leq 0.01$; ${ }^{* * *} P \leq 0.001 ;{ }^{* * * *} P \leq 0.0001$

believed that approximately $40 \%$ of these neurons are sensitive to the static level of BP, while $60 \%$ react to the actual change in BP [24]. This hypothesis was confirmed by Zhang et al., who identified monosynaptic neurons reacting to acute BP changes with a high firing rate of up to $70 \mathrm{~Hz}$, while polysynaptic neurons are activated at a lower frequency of approximately $20 \mathrm{~Hz}$ when a BP level is reached [25]. While studies conducted in isolated perfused carotid sinuses have revealed an increase in baroreceptor activity (BRA) when a static high BP is simulated, this increased activity leads to the attenuation of baroreflex-mediated sympathoinhibition and is, therefore, less efficient in reducing BP [10, 11]. Tanaka et al. even postulated that switching from pulsatile to non-pulsatile 
stimulation may increase BP due to reduced sympathoinhibition via non-pulsatile stimulation [26]. While these studies indicate that pulsatile BRA is more efficient in reducing $\mathrm{BP}$, it has never been investigated whether pulsatile ADN stimulation synchronized to the cardiac cycle allows a slow, but continuous reduction of $\mathrm{BP}$, without causing relevant side effects.

Our results show that energy-efficient selective pulsatile stimulation of the vagal nerve is nearly free of side effects, can lower the BP and keep it continuously at a desired level.

Limitations of the study: the experiments were performed in six animals. Future studies should include a higher number of animals to consolidate the statistical conclusions. Future studies we plan to perform should also elucidate whether the aspects of this study can be directly transferred to larger animals and humans.

\section{CONCLUSION}

CssVNS can continuously reduce BP in male Wistar rats without altering $\mathrm{HR}$ or the $\mathrm{PQ}$ interval. Changing the interval from stimulation at every pulse to every second or third pulse allows BP to be smoothly adjusted to the desired plateau value. CssVNS is, therefore, a promising and energy-efficient technique that might be implemented using antihypertensive selective vagal nerve stimulators in the future.

\section{ACKNOWLEDGMENTS}

The study and article processing charges were funded by Bundesministerium fur Bildung und Forschung/German Federal Ministry of Education and Research among the call "Individualisierte Medizintechnik" under the grant number FKZ 13GW0120B. All named authors meet the International Committee of Medical Journal Editors (ICMJE) criteria for authorship of this manuscript, take responsibility for the integrity of the work as a whole and have given final approval to the version to be published.

Disclosures. Dennis T. T. Plachta is a co-founder of Neuroloop GmbH. Thomas Stieglitz is a co-founder of Neuroloop GmbH. Josef Zentner is a co-founder of Neuroloop $\mathrm{GmbH}$. Mortimer Gierthmuehlen is a co-founder of Neuroloop GmbH. Oscar Cota is an employee of Neuroloop GmbH. Debora Aguirre has nothing to disclose.

Compliance with Ethics Guidelines. This study was approved by the Regierungspraesidium Freiburg, Baden-Wuerttemberg, and the Ethics Committee of the University of Freiburg (G13-44). All institutional and national guidelines for the care and use of laboratory animals were followed.

\section{REFERENCES}

1. Calhoun DA, Jones D, Textor S, Goff DC, Murphy TP, Toto RD, et al. Resistant hypertension: diagnosis, evaluation, and treatment: a scientific statement from the American Heart Association Professional Education Committee of the Council for High Blood Pressure Research. Circulation. 2008;117(25):e510-26.

2. Persell SD. Prevalence of resistant hypertension in the United States, 2003-2008. Hypertension. 2011;57(6):1076-80.

3. Urban D, Ewen S, Ukena C, Linz D, Bohm M, Mahfoud F. Treating resistant hypertension: role of renal denervation. Integr Blood Press Control. 2013;6:119-28.

4. Alnima T, de Leeuw PW, Kroon AA. Baroreflex activation therapy for the treatment of 
drug-resistant hypertension: new developments. Cardiol Res Pract. 2012;2012:587194.

5. Bakris GL, Townsend RR, Liu M, Cohen SA, D'Agostino R, Flack JM, et al. Impact of renal denervation on 24-hour ambulatory blood pressure: results from SYMPLICITY HTN-3. J Am Coll Cardiol. 2014;64(11):1071-8.

6. Bloch MJ, Basile JN. The Rheos Pivotol trial evaluating baroreflex activation therapy fails to meet efficacy and safety end points in resistant hypertension: back to the drawing board. J Clin Hypertens (Greenwich). 2012;14(3):184-6.

7. Bisognano JD, Bakris G, Nadim MK, Sanchez L, Kroon AA, Schafer J, et al. Baroreflex activation therapy lowers blood pressure in patients with resistant hypertension: results from the double-blind, randomized, placebo-controlled rheos pivotal trial. J Am Coll Cardiol. 2011;58(7): 765-73.

8. Durand MT, Mota AL, Barale AR, Castania JA, Fazan $\mathrm{R}$ Jr, Salgado HC. Time course of the hemodynamic responses to aortic depressor nerve stimulation in conscious spontaneously hypertensive rats. Braz J Med Biol Res. 2012;45(5):444-9.

9. Plachta DT, Gierthmuehlen M, Cota O, Espinosa $\mathrm{N}$, Boeser F, Herrera TC, et al. Blood pressure control with selective vagal nerve stimulation and minimal side effects. J Neural Eng. 2014;11(3): 036011.

10. Chapleau MW, Hajduczok G, Abboud FM. Pulsatile activation of baroreceptors causes central facilitation of baroreflex. Am J Physiol. 1989;256(6 Pt 2):H1735-41.

11. Chapleau MW, Abboud FM. Contrasting effects of static and pulsatile pressure on carotid baroreceptor activity in dogs. Circ Res. 1987;61(5):648-58.

12. Gierthmuehlen M, Plachta DT. Effect of selective vagal nerve stimulation on blood pressure, heart rate and respiratory rate in rats under metoprolol medication. Hypertens Res. 2016;39(2):79-87. doi:10.1038/hr.2015.122.

13. Waynforth HB, Flecknell PA. Experimental and Surgical Technique in the Rat. 2nd ed. Amsterdam: Elsevier; 1992.

14. Stieglitz T, Beutel H, Schuettler M, Meyer J-U. Micromachined, polyimide-based devices for flexible neural interfaces. Biomed Microdevices. 2000;2(4):283-94.
15. Zariffa J, Popovic MR. Influence of the number and location of recording contacts on the selectivity of a nerve cuff electrode. IEEE Trans Bio-Med Eng. 2009;17(5):420-7.

16. Plachta DT, Espinosa N, Gierthmuehlen M, Cota O, Herrera TC, Stieglitz T. Detection of baroreceptor activity in rat vagal nerve recording using a multi-channel cuff-electrode and real-time coherent averaging. Conf Proc IEEE Eng Med Biol Soc. 2012;2012:3416-9.

17. Burnier M, Wuerzner G, Struijker-Boudier $H$, Urquhart J. Measuring, analyzing, and managing drug adherence in resistant hypertension. Hypertension. 2013;62(2):218-25.

18. Persell SD, Denecke-Dattalo TA, Dunham DP, Baker DW. Patient-directed intervention versus clinician reminders alone to improve aspirin use in diabetes: a cluster randomized trial. Jt Comm J Qual Patient Saf. 2008;34(2):98-105.

19. Bilgutay A, Lillehei C. Treatment of hypertension with an implantable electronic device. JAMA. 1965;191:649-53.

20. Bilgutay AM, Lillehei CW. Surgical treatment of hypertension with reference to baropacing. Am J Cardiol. 1966;17(5):663-7.

21. Neistadt A, Schwartz S. Effects of electrical stimulation of the carotid sinus nerve in reversal of experimentally induced hypertension. Surgery. 1967;61:923-31.

22. Ramani R. Vagus Nerve Stimulation Therapy for Seizures. J Neurosurg Anesthesiol. 2008;20(1):29-35.

23. Gierthmuehlen M, Aguirre D, Cota O, Zentner J, Stieglitz T, Plachta DTT. Haemodynamic responses to selective vagal nerve stimulation under enalapril medication in rats. PloS One. 2016;11(1):e0147045. doi:10.1371/journal.pone.0147045.

24. Rogers RF, Paton JF, Schwaber JS. NTS neuronal responses to arterial pressure and pressure changes in the rat. Am J Physiol. 1993;265(6 Pt 2):R1355-68.

25. Zhang J, Mifflin SW. Responses of aortic depressor nerve-evoked neurones in rat nucleus of the solitary tract to changes in blood pressure. J Physiol. 2000;529(Pt 2):431-43.

26. Tanaka K, Yada I. NTS neuronal response to conversion from pulsatile to nonpulsatile pressure in isolated carotid sinus baroreceptors. Artif Organs. 2003;27(9):833-9. 